\title{
Correction to: Sex-dependent association between selenium status and cognitive performance in older adults
}

\author{
Barbara Rita Cardoso ${ }^{1}$ (D . Dominic J. Hare ${ }^{2,3} \cdot$ Helen Macpherson ${ }^{4}$
}

Published online: 5 January 2021

c) Springer-Verlag GmbH Germany, part of Springer Nature 2020

\section{Correction to: European Journal of Nutrition}

$$
\text { https://doi.org/10.1007/s00394-020-02384-0 }
$$

Unfortunately, the given name and family name of the author Barbara Rita Cardoso was incorrectly tagged in the xml data, therefore it is abbreviated wrongly as " $\mathrm{R}$ Cardoso B" in Pubmed. The correct given name is Barbara Rita and family name is Cardoso.

The original article can be found online at https://doi.org/10.1007/ s00394-020-02384-0.

Barbara Rita Cardoso

barbara.cardoso@monash.edu

1 Department of Nutrition, Dietetics and Food, Monash University, 264 Ferntree Gully Road, Notting Hill, VIC 3168, Australia

2 School of BioSciences, University of Melbourne, Melbourne, VIC, Australia

3 The Peter Doherty Institute for Infection and Immunity, University of Melbourne, Melbourne, VIC, Australia

4 Institute for Physical Activity and Nutrition (IPAN), Deakin University, Geelong, VIC, Australia 